\title{
Parálisis facial bilateral secundaria a infección por virus de Epstein-Barr
}

\section{Bilateral facial palsy due to Epstein-Barr virus infection}

\author{
M.E. Erro ${ }^{1}$, J. Urriza ${ }^{2}$, L. Gila ${ }^{2}$, E. Orbara ${ }^{3}$ y I. G. de Gurtubay ${ }^{2}$
}

\section{RESUMEN}

Nuestro objetivo es describir dos pacientes jóvenes con parálisis facial periférica bilateral. Ambos presentaron inicialmente afectación en un lado de la cara, seguida pocos días después de afectación contralateral junto con sintomatología compatible con infección aguda por el virus de Epstein-Barr, que se confirmó con la serología. Uno de los pacientes experimentó mejoría completa mientras que en el otro la recuperación fue lenta y quedaron secuelas permanentes. La lesión bilateral del nervio facial es una complicación infrecuente de la infección por el virus de Epstein-Barr cuya evolución no siempre es favorable. Se discute su mecanismo patogénico.

Palabras clave. Diplejia facial. Mononucleosis infecciosa. Reflejo de parpadeo.

\begin{abstract}
Two young patients with bilateral facial palsy are described. They initially presented unilateral facial palsy, followed by contralateral facial nerve involvement a few days later, together with clinical and serologic evidence of acute Epstein-Barr virus infection. The outcome was favourable in one patient but severe sequels persisted in the second. These two cases show that this infrequent complication of Epstein-Barr virus infection may not always have a good outcome. The pathogenic mechanism of bilateral facial palsy is discussed.
\end{abstract}

Key words. Facial diplegia. Infectious mononucleosis. Blink reflex.
1. Servicio de Neurología. Hospital de Navarra. Pamplona.

2. Servicio de Neurofisiología Clínica. Hospital Virgen del Camino. Pamplona.

3. Medicina Familiar y Comunitaria. Centro de Salud de Chantrea. Pamplona.

Recepción: 3 de septiembre de 2009

Aceptación provisional: 19 de enero de 2010

Aceptación definitiva: 1 de febrero de 2010

\section{Correspondencia}

María Elena Erro Aguirre

Servicio de Neurología

Hospital de Navarra

Irunlarrea, 3

31008 Pamplona. Navarra. España

Tfno. 34-848422292

Fax 34-848422303

E-mail: elena.erro.aguirre@cfnavarra.es 


\section{INTRODUCCIÓN}

La parálisis facial bilateral (PFB) supone del 0,3 al $2 \%$ del total de las parálisis faciales $^{1,2}$. Puede definirse como la afectación simultánea de ambos nervios faciales o la sucesión de parálisis facial contralateral en un plazo inferior a 30 días tras el comienzo de la parálisis en el primer lado ${ }^{2}$. Las causas de PFB son muy variadas e incluyen el síndrome de Guillain-Barré ${ }^{3}$, la sarcoidosis ${ }^{4}$, la enfermedad de Lyme $^{5}$ y otros procesos infecciosos como la poliomielitis ${ }^{1}$, la sífilis ${ }^{6}$ o el virus de la inmunodeficiencia humana $(\mathrm{VIH})^{7}$, enfermedades metabólicas como la diabetes, autoinmunes como la granulomatosis de Wegener ${ }^{8}$ y neoplásicas como la leucemia ${ }^{1}$. La PFB es manifestación de una enfermedad sistémica con mucha más frecuencia que la parálisis facial unilateral.

La PFB puede ser también una muy rara complicación de la infección por el virus Epstein-Barr (VEB) ${ }^{9-11}$, virus herpes linfotrópico que puede afectar a distintas partes del sistema nervioso ${ }^{12}$. Describimos dos pacientes con infección por VEB y PFB que tuvieron una evolución diferente.

\section{CASO CLÍNICO}

\section{Paciente 1}

Varón de 25 años que acudió a urgencias por una parálisis facial derecha precedida de dolor faríngeo. El examen físico mostró una tumefacción de las amígdalas con exudado purulento. En el hemograma se apreció una leve leucocitosis $\left(13,9 \times 10^{9} / \mathrm{L}\right)$ con linfocitos atípicos sugestivos de mononucleosis infecciosa. La bioquímica sanguínea fue normal a excepción de una moderada elevación de transaminasas (AST $21 \mathrm{U} / \mathrm{L}$, normal 0-18; ALT 99 U/L normal 0-22). Se comenzó tratamiento con prednisona a las 24 horas de iniciada la parálisis facial y una semana después el paciente vino de nuevo a urgencias por parálisis facial izquierda. En la exploración se apreciaron adenopatías cervicales, hepatomegalia leve y PFB, más grave en el lado derecho. El resto de pares craneales eran normales así como la fuerza muscular en extremidades y los reflejos osteotendinosos. En el hemograma persistía una leve leucocitosis $\left(13,4 \times 10^{9} / \mathrm{L}\right)$ mientras que las enzimas hepáticas se habían normalizado. La radiografía de tórax era normal. Se demostró la presencia de anticuerpos IgM contra el antígeno de la cápside viral (ACV) del VEB por la técnica ELISA. Los anticuerpos antinucleares, las serologías de Borrelia burgdorferi, VDRL, hepatitis B y $\mathrm{C}$, VIH y citomegalovirus (CMV) fueron normales. El líquido cefalorraquídeo (LCR) mostraba ligera pleocitosis (8 leucocitos por $\mathrm{mm}^{3}$ ) con glucosa y proteínas normales. La reacción de polimerasa en cadena (PCR) para el ADN del VEB fue negativa. La tomografía computarizada (TC) craneal y la resonancia magnética (RM) craneal fueron normales. En el estudio neurofisiológico (Tabla 1), realizado a los 14 días del inicio de la parálisis facial derecha, el potencial de acción muscular compuesto (PAMC) del nervio facial derecho registrado sobre el orbicularis oculi mostraba aumento de latencia $(4,2 \mathrm{~ms}$, normal $<4 \mathrm{~ms})$ y reducción de la amplitud $(0,6 \mathrm{mV}$, normal $>1,5$ $\mathrm{mV}$ ). La amplitud del potencial motor del nervio facial izquierdo sobre orbicularis oculi estaba ligeramente disminuida $(1,2 \mathrm{mV})$. En el estudio del reflejo de parpadeo las respuestas dependientes del nervio facial derecho estaban alteradas: R1 ausente y R2 ipsilateral retrasada $(40,9 \mathrm{~ms}$, nor$\mathrm{mal}<38 \mathrm{~ms}$ ) tras estímulo en nervio supraorbitario derecho y $\mathrm{R} 2$ contralateral retrasada $(38,9 \mathrm{~ms}$ frente a $32,7 \mathrm{~ms}$ en el lado contrario, diferencia normal $<5 \mathrm{~ms}$ ) tras estímulo en nervio supraorbitario izquierdo. El electromiograma (EMG) de los músculos faciales no mostraba actividad espontánea de denervación y el patrón de reclutamiento era pobre. El estudio de conducción nerviosa (ECN) de los nervios hipoglosos, así como el EMG, ECN y las ondas F en extremidades fueron normales. Dos meses después la parálisis facial se había resuelto en ambos lados y el estudio neurofisiológico de control fue normal.

\section{Paciente 2}

Varón de 22 años que ingresó por fiebre de $38,2^{\circ} \mathrm{C}$, dolor de faríngeo y malestar. En la exploración presenta una amigdalitis exudativa y adenopatías cervicales. El hemograma mostró leucocitosis $\left(21,7 \times 10^{9} / \mathrm{L}\right)$ con lifocitosis $(60 \%$, normal 20-45) y linfocitos atípicos. La bioquímica sanguínea mostró elevación de transaminasas (AST $86 \mathrm{U} / \mathrm{L}$, normal 0-18; ALT $208 \mathrm{U} / \mathrm{L}$ normal $0-22)$ y lactato deshidrogenada (1187 U/L, normal 240-480). Se estableció el diagnóstico de mononucleosis infecciosa por VEB y se recomendó tratamiento con paracetamol. Cinco días más tarde re-ingresa por una parálisis facial derecha y se inicia tratamiento con prednisona. Una semana después desarrolla una parálisis facial izquierda mostrando una grave PFB en la exploración mientras que la fuerza en extremidades y los reflejos osteotendinosos eran normales. La radiografía de tórax era normal. La TC y 


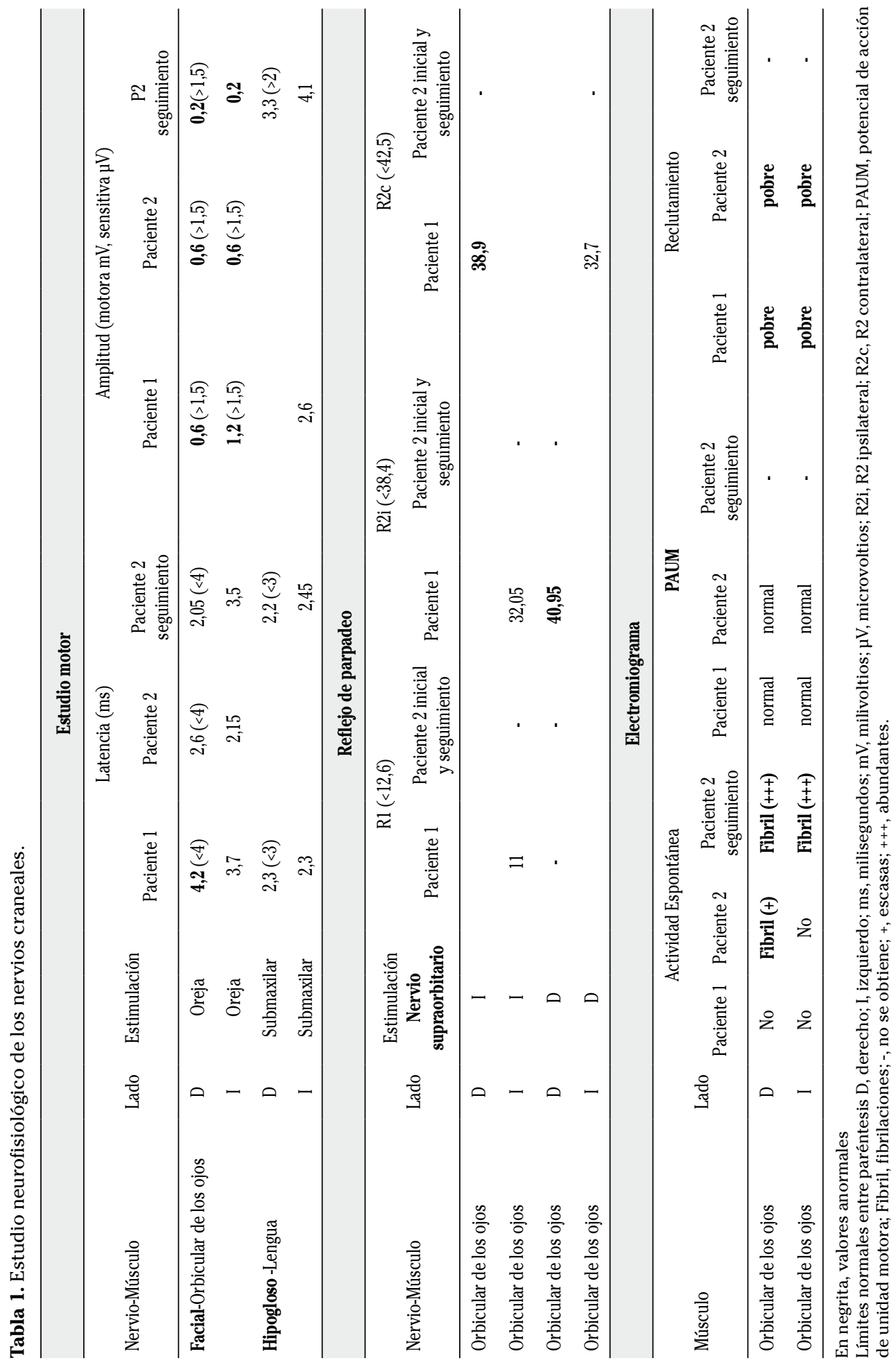


la RM craneal fueron normales. Las pruebas de función hepática se normalizaron a los quince días del inicio de los síntomas. Los estudios serológicos fueron diagnósticos de una infección aguda por el VEB por el hallazgo de anticuerpos positivos IgM contra el VCA del VEB determinado por la técnica de ELISA. Las serologías a Borrelia burgdorferi Lyme, hepatitis A, B y C, VIH y CMV fueron negativas. El análisis del LCR mostró una plecitosis linfocitaria con 30 células por $\mathrm{mm}^{3}$ y contenido de glucosa y proteínas normales. La PCR en LCR para el ADN del VEB fue negativa. En el ECN realizado a los 11 días de la primera parálisis facial, la amplitud de los potenciales motores de ambos nervios faciales estaba muy disminuida $(0,6 \mathrm{mV})$. No se obtuvieron respuestas en el estudio del reflejo de parpadeo. El EMG de los músculos orbicularis oculi mostró escasas fibrilaciones en el lado derecho y un patrón de reclutamiento pobre en ambos lados con potenciales de unidad motora (PAUMs) normales (Tabla 1). El EMG, ECN y ondas F en extremidades fueron normales. Se instauró tratamiento con corticoides orales durante un mes. Un estudio neurofisiológico de control realizado un mes después mostró PAMC de los nervios faciales con registro en los músculos orbicularis oculi de muy baja amplitud $(0,2 \mathrm{mV})$ y ausencia de respuesta en el estudio del reflejo de parpadeo. En el EMG de estos músculos se encontró abundante actividad espontánea de denervación en forma de fibrilaciones y ondas agudas positivas y no se registraron PAUMs. El examen neurológico a los 6 meses del inicio de los síntomas no mostraba mejoría significativa pero un año después se constató una recuperación importante de manera que el paciente conseguía ocluir los párpados con ligera debilidad y movilizar la musculatura facial inferior bilateralmente con leve espasticidad y debilidad residual.

\section{DISCUSIÓN}

Los dos pacientes presentados corresponden a sendos casos de PFB en el contexto de infección aguda por el VEB cuyo diagnóstico se estableció por las manifestaciones clínicas típicas de esta entidad y por los hallazgos serológicos. Las complicaciones neurológicas debidas a la infección por el VEB incluyen encefalitis aguda, mielitis, neuritis óptica ${ }^{12}$ y diversos síndromes periféricos como mononeuritis múltiple, neuropatía sensitiva subaguda ${ }^{13}$, síndrome poliomielítico ${ }^{14}$ y radiculopatías cervicales y lumbares ${ }^{15-16}$. Las parálisis reversibles de varios pares craneales se han descrito sobre todo en niños afectando a los nervios hipogloso y recurrente laríngeo ${ }^{17-18}$.

Aproximadamente el $40 \%$ de las PFB asociadas al VEB son bilaterales y en la mayoría de los casos descritos en la literatura la evolución es favorable con recuperación rápida y completa ${ }^{2}$. La lesión del nervio facial por el VEB puede explicarse por dos mecanismos fisiopatológicos: una infección directa por el virus o una inflamación postinfecciosa inmunomediada. En los dos pacientes que describimos la PFB se produjo coincidiendo con los síntomas de la mononucleosis infecciosa y con pleocitosis linfocitaria en LCR lo que apunta a una lesión directa del nervio facial por el EBV. Se ha descrito infección vírica directa de las células de Schwann en un caso de polineuropatía desmielinizante primaria con daño axonal secundario por $\mathrm{CMV}^{19}$. Respecto al segundo mecanismo, la PFB como parte de una neuropatía disinmune precipitada por el EBV es bien conocida y en estos casos la debilidad facial suele acompañarse de debilidad en extremidades, datos neurofisiológicos de polirradiculopatía o elevación de proteínas en LCR, ausentes todos ellos en los dos casos que presentamos por lo que el mecanismo inmune parece improbable, aunque no pueda ser totalmente excluido dada la existencia de formas regionales del síndrome de Guillain-Barré que cursan exclusivamente con diplejía facial ${ }^{3}$. Por otro lado, teniendo en cuenta que en ambos pacientes la PFB no fue simultánea, cabe la posibilidad de que cada una de las parálisis faciales pudiera deberse a un mecanismo fisiopatológico distinto.

Tanto la infección directa como la reacción disinmune pueden producir daño de la mielina y secundariamente del axón. Junto con estos mecanismos básicos pueden coexistir otros factores que determinen el grado de afectación, como serían las características anatómicas del canal facial. Si las dimensiones de éste son relativamente reducidas, la reacción inflamatoria puede condicionar un daño sobreañadido por compresión del nervio. 
Los hallazgos neurofisiológicos en uno y otro caso son netamente diferentes, correlacionándose con el distinto grado de severidad y forma de evolución de cada uno de ellos. El primer paciente, que experimentó una resolución completa de la PFB, mostraba una latencia aumentada del PAMC en uno de los nervios faciales y ausencia de actividad espontánea de denervación en la EMG, lo que sugiere una afectación desmielinizante. Sin embargo, en el segundo paciente las alteraciones neurofisiológicas corresponden a un patrón de afectación axonal, con abundante actividad espontánea en la EMG y latencias normales de los PAMC, congruente con su peor evolución.

En conclusión, los casos descritos ilustran el espectro clínico en cuanto a grado de afectación y forma evolutiva de una rara complicación neurológica de la infección por VEB como es la PFB. Presumiblemente, el mecanismo patogénico por infección vírica directa de los nervios faciales es el mismo en ambos casos, pero con muy diferentes consecuencias respecto a forma predominante de lesión, desmielinizante o axonal y pronóstico correspondiente.

\section{BIBLIOGRAFÍA}

1. KEANE JR. Bilateral seventh nerve palsy. Neurology 1994; 44: 1198-1202.

2. Teller DC, Murphy TP. Bilateral facial palsy: a case presentation and literature review. J Otolaryngol 1992: 21: 44-47.

3. Susuki K, Atsumi M, Koga M, HiRata K, Yuki $\mathrm{N}$. Acute facial diplegia and hyperreflexia. A Guillain-Barré syndrome variant. Neurology 2004; 62: 825-827.

4. Jain V, Deshmukh A, Gollomp S. Bilateral facial paralysis. Case presentation and discussion of differential diagnosis. J Gen Intern Med 2006; 21: 7-10.

5. Gevers G, Lamkens P. Bilateral simultaneous facial paralysis-differential diagnosis and treatment options. A case report and review of the literature. Acta Otorhinolaryngol Belg 2003; 57: 139-146.
6. Del Río C, Aguilera JM, Cabrera M, Romero B, Romera M, Fernández-Bolaños R. Diplejía facial como inicio de una neurosífilis. Neurología 2007; 22: 745.

7. Gállego Pérez-Larraya J, González R, Gómez A, Fernández R, Riverol M. Diplejia facial y neuritis vestibular como signo de primoinfección por VIH. Neurología 2007; 22: 720.

8. Nikolaou AC, Vlachtsis KC, Danilidis MA, PETRIDIS DG, DANIILIDIS IC. Wegener's granulomatosis presenting with bilateral facial nerve palsy. Eur Arch Otorhinolaryngol 2001; 258: 198-202.

9. Diedler J, Rieger S, Koch A, Parthé-PeterHANs, Schwaninger M. Bilateral facial palsy: Epstein-Barr virus, not Lyme disease. Eur J Neurol 2006; 13: 1029-1030.

10. Terada K, Nizuma T, Kosaka Y, Inoue M, Ogita S, Kataoka N. Bilateral facial nerve palsy associated with Epstein-Barr virus infection with a review of the literature. Scand J Infect Dis 2004; 36: 75-77.

11. Bechich S, Salas R, Arboix A. Facial dyplegia by Epstein-Barr virus. Med Clin (Barcelona) 1998; 110: 519-520.

12. Fujimoto H, Asaoka K, Imaizumi T, Ayabe M, SнолI H, KaJI M. Epstein-Barr virus infections of the central nervous system. Internal Medicine 2003; 42: 33-40.

13. Rubin DI, DAUBE JR. Subacute sensory neuropathy associated with EpsteinBarr virus. Muscle Nerve 1999; 22: 16071610.

14. Wong M, Connolly AM, Noetzel MJ. Poliomyelitis-like syndrome associated with Epstein-Barr virus infection. Pediatric. Neurology 1999; 20: 235-237.

15. Pou Serradell A, Royo I, Aragones JM, Zapater E, Panella A. Painful bilateral lumbar radiculoplexopathy associated with Epstein-Barr virus infection. Rev Neurol (Paris) 1996; 152: 483-485.

16. Vucic S, PALmer W, CRos D. Radiculoplexopathy with conduction block caused by acute Epstein-Barr virus infection. Neurology 2005; 64: 530-532.

17. Parano E, Pavone L, Musumeci S, Giambusso F, TRIFLLETTI RR. Acute palsy of the re- 
current laryngeal nerve complicating Epstein-Barr virus infection. Neuropediatrics 1996; 27: 164-166.

18. Parano E, Giuffrida S, Restivo D, Saponara R, GRECO F, TRIFILETTI R. Reversible palsy of the hypoglossal nerve complicating infectious mononucleosis in a young child. Neuropediatrics 1998; 29: 46-47.

19. Morgello S, Simpson DM. Multifocal cytomegalovirus demyelinative polyneuropathy associated with AIDS. Muscle Nerve 1994; 17: 176-182. 